Results: In total, 22 and 53 patients were assigned to BRCA mutation and wild-type groups, respectively. Between the 2 groups, no differences in patient age, proportions of highgrade serous carcinoma and bevacizumab users, and the use of maintenance therapy were observed. However, the BRCA mutation group had a significantly longer platinum-free interval ( $\mathrm{p}=0.021)$ and a higher rate of complete cytoreduction with borderline statistical significance ( $95.5 \%$ vs. $73.6 \%$; $\mathrm{p}=0.053)$, compared to the BRCA wild-type group. Of 22 patients in the BRCA mutation group, 8 (36.4\%) received PARP inhibitor maintenance therapy after completion of second-line platinum-based combination chemotherapy. After a median follow-up of 38.2 months, the groups showed similar overall survival; however, the BRCA mutation group displayed better progression-free survival (PFS; median, 28.4 vs. 18.4 months; $\mathrm{p}=0.012$ ). Multivariate analyses identified BRCA1/2 mutation as an independent favorable prognostic factor for PFS (adjusted hazard ratio $=0.419 ; 95 \%$ confidence interval $=0.218-0.806$; $\mathrm{p}=0.009)$. Consistent results were observed among 60 patients who achieved complete cytoreduction.

Conclusion: In the case of secondary cytoreductive surgery for PSR EOC, patients with BRCA1/2 mutations have a better prognosis with longer PFS than those lacking BRCA mutations. Further studies with a large sample size are warranted to confirm our findings.

Oral (006)

Epithelial Ovarian Cancer including Borderline Tumor

https://doi.org/10.3802/jgo.2021.32.S1.006

\section{Clinical characteristics of early-stage clear cell ovarian cancer: a 10-year retrospective experience in Ren Ji Hospital}

\section{Xiaoshi Liu, Wen $\mathrm{Di}^{*}$}

Ren Ji Hospital, School of Medicine, Shanghai Jiao Tong University, Shanghai, China (diwen163@163.com)

Objective: Ovarian clear cell carcinoma (OCCC) is a distinct subtype of epithelial ovarian cancer with unique clinical and molecular characteristics. It is likely to be found at an earlystage and its prognosis is relatively good. However, it has recently been reported that in early-stage OCCC, some recur more frequently and become chemoresistant according to subtypes, due to occult metastasis. This study was conducted to learn more about the clinical characteristics and outcomes of early-stage OCCC, as well as to provide more information to help with clinical diagnosis and therapy.

Methods: The clinical features such as age, preoperative cancer antigen 125 (CA125), CA19-9, platelet, plasma D-dimer, systemic inflammatory response (SIR) markers, treatment and survival outcomes of 70 patients with International Federation of Gynecology and Obstetrics I-II OCCC treated at our center between August 2010 and April 2020 were investigated in this retrospective analysis. Initially, receiver operating characteristic curve analysis for overall survival (OS) was used to determine optimal cut-off values for preoperative parameters $(\mathrm{CA} 125=52.65$, CA19-9 $=21.3$, platelet $=223.5$, SIR index [SIRI]=1.289). Kaplan-Meier curves were used for the analysis of OS and progression-free survival (PFS). The p-values were calculated by a log-rank test. Multivariate Coxregression analysis was used to further assess the variables proved significant in univariate analysis. $\mathrm{p}<0.05$ was considered statistically significant.

Results: Overall, the mean age of participants was $57.46 \pm 9.41$ years old. All patients underwent hysterectomy with bilateral salpingo-oophorectomy, and $87.1 \%$ of them underwent lymphadenectomy. Of the 62 women with available chemotherapy data, $42(67.7 \%)$ received one cycle intraperitoneal chemotherapy with cisplatin and 59 (95.2\%) underwent more than 3 cycles of chemotherapy. The 48 patients received open procedures and the rest underwent laparoscopy. There were 14 recurrences and 3 deaths during the median follow-up duration of 55.2 months. The median CA125 level was 56.27 IU/mL (range 5.81-602.8 IU/mL), with normal CA125 levels seen in 20 patients. Almost three quarters of patients (77.1\%) assayed had a normal CA19-9, with a median level of 15.6 IU/mL. In this study, no statistically significant correlations between the differential pretreatment levels of CA125 $(\mathrm{p}=0.87$ for OS, $\mathrm{p}=0.71$ for PFS)/CA19-9 $(\mathrm{p}=0.05$ for OS, $\mathrm{p}=0.95$ for PFS) and clinical outcomes in patients with early-stage OCCC were found. SIRI $(p=0.008)$ and platelet $(p=0.002)$ levels were identified as prognostic factors for OS. SIRI $(p=0.024)$ was also prognostic factor for PFS. Patients who received one cycle intraperitoneal chemotherapy were related to an obviously better prognosis ( $\mathrm{p}=0.032$ for OS, $\mathrm{p}=0.048$ for PFS). Furthermore, multivariate analysis showed that an increase in SIRI is a significant independent prognostic factor for poor prognosis (hazard ratio=3.192, $\mathrm{p}=0.00215$ ).

Conclusion: Early-stage OCCC often presents with mildly elevated CA125 and adding one cycle intraperitoneal chemotherapy can offer additional survival benefit to patients. Pretreatment evaluated SIRI might be potential biomarkers for worse response to first-line chemotherapy and poorer clinical outcomes. Therefore, more specific treatment strategies and a rigorous follow-up plan can be formulated for patients with early-stage OCCC based on clinical characteristics and preoperative parameters. 